\title{
Epilepsy
}

\section{Five new things}

Chutima Saipetch, MD*; Ezekiel Sachs, MD; Zulfi Haneef, MD*

\begin{abstract}
Purpose of review: Technological advance has revolutionized epilepsy management recently. Herein, we review some recent developments. Recent findings: Responsive neurostimulation (Food and Drug Administration [FDA]-approved 2013) works by continuous analysis of brain rhythms and direct brain stimulation on detecting patterns thought to be epileptogenic, thereby aborting seizures. Cardioresponsive vagus nerve stimulation (FDA-approved 2015) is an improvement over traditional vagus nerve stimulation systems, taking advantage of the fact that $80 \%$ of seizures are associated with tachycardia. Automated tachycardia detection leads to vagus nerve stimulation to abort seizures. In MRIguided stereotactic laser ablation (developed
\end{abstract}

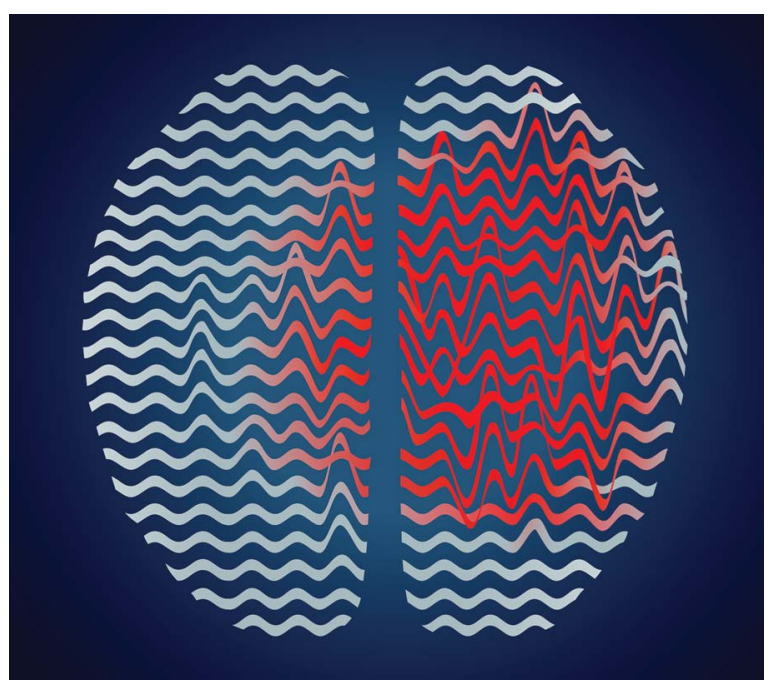
2012), a directed laser emitting fiberoptic catheter is used to ablate epileptogenic lesions. The procedure can be completed in 3 to 4 hours, potentially under local anesthesia and with next-day discharge. Perampanel (FDA-approved 2012) is a promising new class of AMPA ( $\alpha$-amino-3-hydroxy-5-methyl-4-isoxazole propionic acid)-antagonist antiseizure therapy. Meanwhile, a millennia-old remedy for epilepsy, cannabis, is staging a comeback with recent legal and social permissiveness accelerating research into this use. Summary: The coming years will demonstrate how these recent advances in device and drug management will improve the care of epilepsy. Neurol Clin Pract 2016;6:444-451

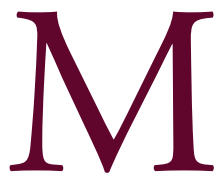

edically refractory epilepsy occurs in $30 \%$ of patients with epilepsy. Among such patients, those with an identifiable epileptogenic lesion are candidates for surgical remediation, traditionally by open resection. ${ }^{1}$ Technological advances and drug development have resulted in several new and potentially game-changing innovations to help treat such patients. Herein, we review 5 such innovations, examining how these new devices and drugs work, and the research that underlies the promise offered by these to patients with epilepsy.

\footnotetext{
*These authors contributed equally to this work.

Baylor College of Medicine (CS), Houston, TX; Emory University (ES), Atlanta, GA; and Baylor College of Medicine and the VA Medical Center ( $\mathrm{ZH})$, Houston, TX.

Funding information and disclosures are provided at the end of the article. Full disclosure form information provided by the authors is available with the full text of this article at Neurology.org/cp.

Correspondence to: zulfi.haneef@bcm.edu
} 


\section{Minimally invasive alternatives to open} resection include stereotactic radiosurgery, radiofrequency ablation, and most recently magnetic resonance-guided stereotactic laser ablation.

\section{Magnetic resonance-guided stereotactic laser ablation}

Minimally invasive alternatives to open resection include stereotactic radiosurgery, radiofrequency ablation, and most recently magnetic resonance-guided stereotactic laser ablation (MRGSLA).

First reported in 2012, ${ }^{2}$ MRGSLA has been an area of active research for various epileptic lesions such as hippocampal sclerosis, ${ }^{3}$ cortical dysplasia, tuberous sclerosis, periventricular nodular heterotopia, ${ }^{4}$ hypothalamic hamartoma, ${ }^{5}$ cerebral cavernous malformations, ${ }^{6}$ CNS neoplasms, ${ }^{7}$ and radiation necrosis. ${ }^{2}$ In a case series of 13 patients who underwent MRGSLA for mesial temporal lobe epilepsy, 8 were free of disabling seizures at 1 year, and 6 of 9 patients with mesial temporal sclerosis were seizure-free at 1 year. Potential complications reported included subdural hematoma and visual field deficit. ${ }^{8}$

The procedure involves positioning the patient's head within a stereotactic frame and guiding a laser emitting fiberoptic catheter through an anchor bolt to the surgical target. The laser diffuser delivers thermal energy, and magnetic resonance thermography is used to monitor the tissue temperature and to calculate the irreversible ablation volume with precision. ${ }^{9}$ Automated safety points prevent excessive heating and ablation of off-target tissues. Multiple ablations can be made over the length of a single trajectory and multiple trajectories can be used to ablate complex lesions. ${ }^{2}$

Advantages of MRGSLA over other noninvasive modalities include the ability to monitor an otherwise blind surgical procedure in real time, immediate ablation without known delayed effect, the option of not using general anesthesia, and shorter postoperative hospital stay typically without intensive care unit monitoring. ${ }^{3}$ MRGSLA also allows treatment of deep lesions otherwise inoperable without damaging overlying eloquent cortex and white matter tracts. Sparing superficial brain tissues may obviate cognitive deficits encountered after traditional anterior temporal lobectomy. ${ }^{10}$ In addition, patients otherwise hesitant to undergo elective epilepsy surgery may be willing for this less invasive procedure. There is not enough experience to determine definitively the safety and efficacy profile of MRGSLA for epilepsy, although the patient outcomes published to date are encouraging that this could be a comparable alternative to open resection in mesial temporal lobe epilepsy. ${ }^{8}$ Repeat ablation or future resection can also be considered if seizures remain refractory.

\section{Responsive neurostimulation}

Responsive neurostimulation (RNS) (marketed as NeuroPace) is the only Food and Drug Administration (FDA)-approved (2013) responsive closed-loop focal cortical stimulator for the adjunctive treatment of drug-resistant epilepsy. It is approved for patients aged 18 years and older with focal epilepsy and $\leq 2$ epileptogenic foci.

A small battery-operated RNS device is implanted in the patient's skull with leads placed on the cortical surface or within deeper structures thought to be epileptogenic (figure 1). Up to 4 depth and strip electrodes, each with 4 contacts, can be used to target the putative epileptogenic zone(s), although only 2 of the electrodes can be used at one time for recording and stimulation. In principle, RNS is similar to a cardiac automated defibrillator that detects and aborts arrhythmias - it continuously monitors brain waves to detect patterns that can lead to seizures and generates stimuli to abort them. After device implantation, the patient is 
Figure 1 Responsive neurostimulation device

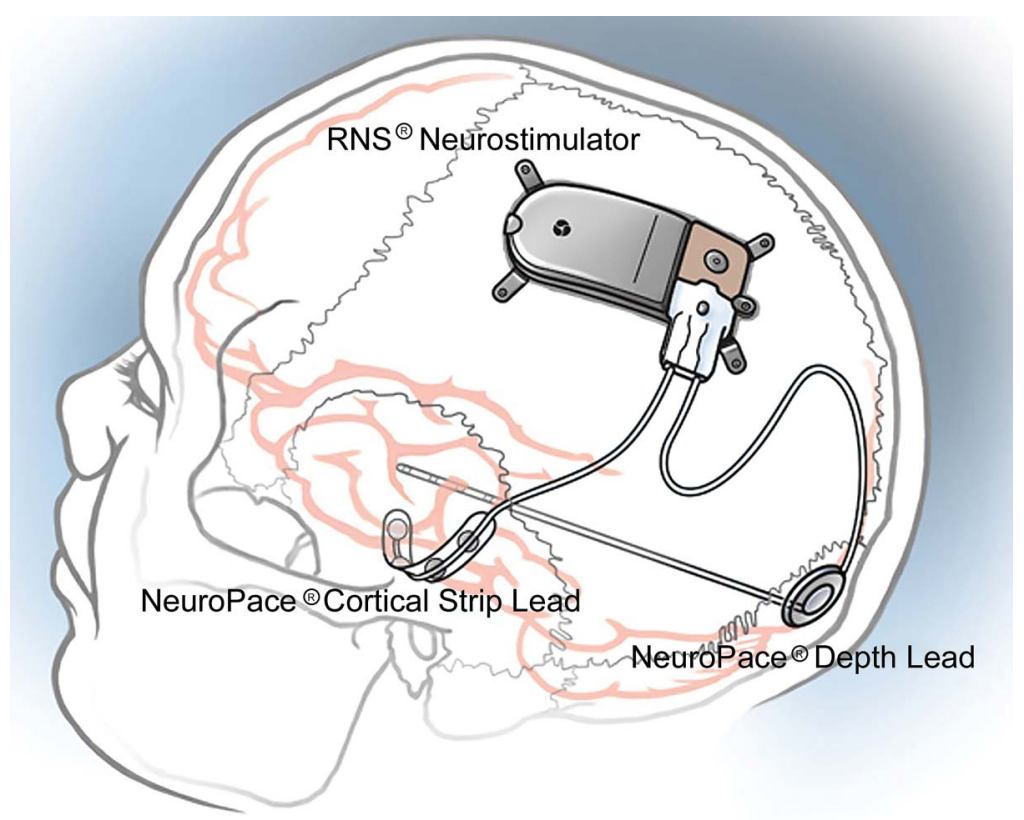

RNS = responsive neurostimulation. Photo courtesy of NeuroPace Inc. @ Copyright 2015.

provided with a system to transfer information recorded to an online patient data management system. The physician accesses this information and identifies electrocorticogram patterns leading to seizures. The device is then programmed to detect this pattern and deliver electrical stimulation to abort seizure onset (figure 2). Detection and stimulation measures can be fine-tuned over time to detect and abort more seizures. ${ }^{11}$

In the pivotal RNS study published in 2011, 191 adults with drug-resistant focal epilepsy reporting $\geq 3$ seizures per month were implanted with RNS. ${ }^{12}$ Subjects were randomized to receive stimulation in response to detections (treatment) or to receive no stimulation (sham). Over a 12-week blinded period, the "treatment" group had a 37.9\% reduction in seizure frequency compared to $17.3 \%$ in the "sham" group $(p=0.012)$.

In a subsequent 84-week open-label period during which all subjects received stimulation, seizure reduction was sustained in the "treatment" group and was significantly reduced in the "sham" group. In a follow-up study published in 2014, ${ }^{11}$ all 191 subjects who received active treatment after the fifth postimplant month were followed for 2 years. The median reduction in seizures was $44 \%$ at 1 year and $53 \%$ at 2 years, representing a progressive and significant improvement over time $(p<0.0001)$. There was no significant difference in adverse event rate between treatment and sham groups. ${ }^{11}$ The median seizure reduction was $48 \%$ to $66 \%$ over postimplant years 3 through $6 .{ }^{13}$ Notable side effects included intracranial hemorrhage (4.7\%), mostly within the first days postimplant, and implant site infection (9\%). However, these were not higher than similar rates associated with intracranial electrode implantation, epilepsy surgery, or with deep brain stimulation for movement disorders. Sustained improvement in the quality of life in areas such as attention, language, memory, seizure worry, work, and social function was also reported.

\section{Cardio-responsive vagus nerve stimulator}

Approximately $80 \%$ of seizures are associated with tachycardia. ${ }^{14}$ In June 2015, the FDA approved a closed-loop cardio-responsive vagus nerve stimulator (crVNS) (marketed as AspireSR by Cyberonics, Houston, TX) that incorporates a customizable cardiac-based seizure detection algorithm (CBSDA) to detect seizure-associated tachycardia and deliver automatic 
Figure 2 Demonstration of seizure detection by the responsive neurostimulation device during an early postimplant visit

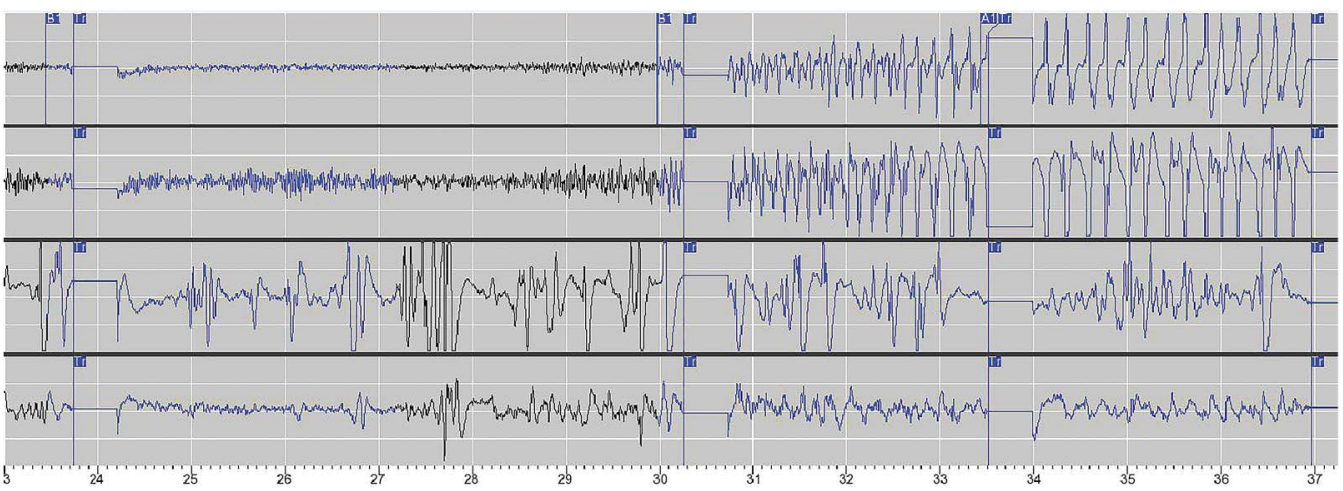

Pattern B1 is detected by the stimulator as potentially epileptic resulting in stimulation (Tr). The pattern is not terminated with this stimulation (B1 detected again), resulting in further stimulation (Tr) to abort a seizure. However, in this instance, the pattern evolves into a fully developed seizure pattern (A1), indicating that further adjustments to the programming may be indicated to improve seizure detection/termination.

stimulation to abort the seizures. This offers an innovative, less-invasive method to detect and treat seizures in real time in selected patients.

The CBSDA tracks heart rate using both long- and short-term heart rate changes. The longterm changes are slow over time based on patient activity. The threshold for seizure detection is programmed to be $20 \%$ to $70 \%$ above this continually adjusted value. When the short-term heart rate change exceeds the threshold, an event is detected and stimulation is delivered.

In a prospective, multicentered study, 31 patients with a history of ictal tachycardia were implanted with crVNS. ${ }^{15}$ Four weeks after implantation, the patients were admitted for 3 to 5 days in the epilepsy monitoring unit. Of 66 seizures analyzed, 37 had a $\geq 20 \%$ increase in heart rate and 11 had ictal tachycardia, defined as a $55 \%$ or $35-\mathrm{bpm}$ increase in heart rate to a minimum of $100 \mathrm{bpm}$. When a patient's relative heart rate increased above a programmed threshold for $\geq 1$ second, a single stimulation train of 30 or 60 seconds was delivered. Patients were randomized to 3 CBSDA settings of $\geq 20 \%$, $\geq 40 \%$, and $\geq 60 \%$ above the baseline heart rate, with which the seizure detection sensitivity was $100 \%, 46.7 \%$, and $27.3 \%$, respectively. With ictal tachycardia, corresponding detection sensitivities were $100 \%, 75 \%$, and $100 \%$. Tachycardia was detected up to 10 seconds before clinical or EEG seizure occurred. False-positive rates per hour were 7.15, 2.72, and 0.49, with CBSDA thresholds of 20\%, $40 \%$, and $60 \%$, respectively. In 27 of 66 seizures, crVNS was triggered within \pm 2 minutes of seizure onset and the seizure terminated during stimulation in 10 of them. The study also showed significant improvement in physician-scored seizure severity for complex partial seizures at epilepsy monitoring unit discharge and through 12 months, as well as patientscored seizure severity at 3 and 6 months, compared to the baseline seizure frequency. There was significant improvement in the quality of life at 12 months. Responder rate $(>50 \%$ reduction in seizure frequency) was $29.6 \%$ at 12 months.

The safety profile for crVNS is comparable to the older VNS models. The most common side effects remain hoarseness, paresthesia, dyspnea, sore throat, and increased coughing. Cardiac-based automated stimulation should not be used in patients with clinically meaningful arrhythmias (e.g., pacemaker dependency), implantable defibrillator, or $\beta$-adrenergic blocker use. Patients also should not have a history of chronotropic incompetence, such as those with sustained bradycardia with heart rate $<50 \mathrm{bpm}$.

While the available data show that tachycardia can be detected by the crVNS device, it is unclear whether there is additional efficacy over earlier-generation VNS devices by virtue of the cardio-responsive feature. A head-to-head comparison of crVNS to older VNS devices is 


\section{The true utility of cannabinoid therapy in epilepsy remains to be determined but it is an area of active academic and public interest.}

not available. However, the device is not markedly more expensive than prior VNS devices, and the cardio-responsive feature can be turned off if not found to be beneficial.

\section{Perampanel}

Perampanel (marketed as Fycompa by Eisai, Woodcliff Lake, NJ) is a highly selective, noncompetitive AMPA ( $\alpha$-amino-3-hydroxy-5-methyl-4-isoxazole propionic acid) receptor antagonist that reduces neuronal hyperexcitability by targeting glutamate activity at postsynaptic AMPA receptors. It is the first AMPA receptor antagonist class of antiepileptic drug to be approved by the FDA. Perampanel is important because of both its unique mechanism of action on AMPA as well as its efficacy against both focal-onset and generalized-onset epilepsy. It is approved as an adjunct therapy for focal-onset seizures and primary generalized tonic-clonic (PGTC) seizures in patients aged 12 years and older. ${ }^{16}$

A pooled analysis of 3 multicenter, randomized, double-blind, placebo-controlled, phase III studies has demonstrated a $23.3 \%$ to $27.2 \%$ reduction in focal-onset seizures (vs $12.8 \%$ for placebo, $p<0.01$ ) when perampanel is used as adjunctive treatment. ${ }^{17}$ In a placebocontrolled clinical phase III study in 164 patients aged 12 years and older with PGTC seizures, a reduction in seizure frequency was observed in the perampanel group compared with placebo $(76.5 \%$ vs $38.4 \%, p<0.0001)$. The responder rate was also higher in the perampanel group than in the placebo group $(64.2 \%$ vs $39.5 \%, p=0.0019)$. Furthermore, $30.9 \%$ of patients treated with perampanel were free of PGTC seizures, compared to $12.3 \%$ in the placebo group during the 13 -week maintenance period. ${ }^{16}$

Perampanel's long half-life of 106 hours allows once-daily dosing. Enzyme-inducing antiepileptic drugs can reduce perampanel plasma concentrations and decrease its efficacy. ${ }^{17}$ The most common adverse events are dizziness, fatigue, headache, and somnolence. Specific adverse effects to monitor are neuropsychiatric events, including aggression, anger, homicidal ideation, hostility, and irritability. These side effects are dose-related and most often occur in the first 6 weeks of therapy. While the novel mechanism of action of perampanel is intriguing, it has to be noted that there have been no head-to-head studies comparing it to other antiseizure medications, and as such, its efficacy compared to currently available medications is not clear.

In addition, one has to be aware of the boxed warning required by the FDA to alert prescribers and patients about the risk of serious neuropsychiatric events, including irritability, aggression, anger, anxiety, paranoia, euphoric mood, agitation, and mental status changes. In the phase 3 clinical trials, hostility- and aggression-related adverse reactions were seen in $12 \%$ of patients receiving perampanel of $8 \mathrm{mg}$ and $20 \%$ of those on $12 \mathrm{mg}$ per day compared to $6 \%$ in the placebo group. These effects were dose-related and typically appeared within the first 6 weeks of treatment. Three of 4,368 perampanel-treated patients exhibited homicidal ideation or threat. The combination of alcohol and perampanel significantly worsened mood and increased anger, and the product labeling recommends avoiding the use of alcohol while on therapy with perampanel. ${ }^{18}$

\section{Cannabidiol}

Cannabis has been used in the treatment of epilepsy through history, perhaps as early as 4000 BC. ${ }^{19}$ Published evidence to support this use has been largely limited to case reports and animal model data until recently, in part limited by its status as a schedule I controlled substance and the biochemical heterogeneity of commercially available cannabis. The 
structure of cannabidiol (CBD) was clarified in $1963,{ }^{20}$ and that of tetrahydrocannabinol (THC) in $1964 .{ }^{21}$ The cannabinoid receptor was demonstrated in $1988 .^{21} \mathrm{THC}$ is the psychoactive component, ${ }^{21}$ while CBD may have anticonvulsive effects. ${ }^{22}$ THC works by partial agonism of central cannabinoid receptor type 1 (CB1) receptors, ${ }^{22}$ which decreases neuronal excitability. CBD's modulation of neuronal excitability is incompletely understood, and it has primary molecular targets distinct from THC. ${ }^{22}$ Considering the above, and the chemical heterogeneity of various cannabis plant strains, it is understandable that there are conflicting reports regarding the effect of cannabis use in seizures. ${ }^{23,24}$

Recent relaxation of the legal restrictions on cannabis use and reports of successful treatment of medically refractory epilepsy with cannabinoids ${ }^{19,22}$ have heightened popular interest in pursuing cannabinoid-based therapies. A Cochrane review in 2014 identified 4 qualifying randomized controlled trials of the efficacy of CBD for epilepsy. While the review reported no significant adverse events with cannabinoid use, it was deemed that no reliable conclusions could be drawn regarding the efficacy of cannabinoids as a treatment for epilepsy. ${ }^{25}$

A recent open-label trial ${ }^{26}$ investigated the safety and efficacy of adjunctive CBD (2-50 $\mathrm{mg} / \mathrm{kg} / \mathrm{d}$ ) in patients aged 30 years and younger with medically refractory childhood-onset epilepsy. In contrast to the previously reviewed studies, $12 \%$ of patients experienced a severe adverse event possibly related to CBD use, including status epilepticus, diarrhea, pneumonia, and weight loss. There was a $36.5 \%$ median reduction of motor seizures over 12 weeks: $39 \%$ had a reduction of $\geq 50 \%, 21 \%$ had a reduction of $\geq 70 \%$, and $4 \%$ were seizure-free. Although this trial was not randomized or controlled, and did not provide the 12-month outcomes sought after in the previously described Cochrane review, 214 patients were enrolled and the results have provided further encouragement for continued research. The study suggests efficacy and a tolerable safety profile for adjunctive CBD in medically refractory epilepsy among young patients.

A recent press release ${ }^{27}$ reports a phase 3 clinical trial of 120 patients with Dravet syndrome randomized to receive CBD $20 \mathrm{mg} / \mathrm{kg} / \mathrm{d}(\mathrm{n}=61)$ and placebo $(\mathrm{n}=59)$, in addition to their current treatment regimen. Comparison of the 14-week treatment period to a 4 -week baseline period showed a median seizure reduction of $30 \%$ with CBD compared to $13 \%$ with placebo $(p=0.01)$. This improvement emerged in the first month of treatment and was sustained during the entire treatment period. Formal publication of the results is awaited.

The true utility of cannabinoid therapy in epilepsy remains to be determined but it is an area of active academic and public interest. There are at least 20 proposed and active trials registered with clinicaltrials.gov to date for CBD in the treatment of epilepsy. It is hoped that this renewed interest will provide the evidence needed to determine whether the benefit of cannabinoids lives up to the promise.

\section{CONCLUSIONS}

Recent advances have resulted in several new therapies for the management of refractory epilepsy. While promising, the available data are not sufficient to conclusively establish these innovations as an improvement over existing therapies. Continuing research will be needed to establish the overall place of these new therapies in epilepsy care. 


\section{REFERENCES}

1. Wiebe S, Blume WT, Girvin JP, Eliasziw M; Effectiveness, Efficiency of Surgery for Temporal Lobe Epilepsy Study Group. A randomized, controlled trial of surgery for temporal-lobe epilepsy. N Engl J Med 2001;345:311-318.

2. Curry DJ, Gowda A, McNichols RJ, Wilfong AA. MR-guided stereotactic laser ablation of epileptogenic foci in children. Epilepsy Behav 2012;24:408-414.

3. Willie JT, Laxpati NG, Drane DL, et al. Real-time magnetic resonance-guided stereotactic laser amygdalohippocampotomy for mesial temporal lobe epilepsy. Neurosurgery 2014;74:569-584; discussion 584-585.

4. Esquenazi Y, Kalamangalam GP, Slater JD, et al. Stereotactic laser ablation of epileptogenic periventricular nodular heterotopia. Epilepsy Res 2014;108:547-554.

5. Rolston JD, Chang EF. Stereotactic laser ablation for hypothalamic hamartoma. Neurosurg Clin N Am 2016;27:59-67.

6. McCracken DJ, Willie JT, Fernald BA, et al. Magnetic resonance thermometry-guided stereotactic laser ablation of cavernous malformations in drug-resistant epilepsy: imaging and clinical results. Neurosurgery Epub 2015 Sep 25.

7. Medvid R, Ruiz A, Komotar RJ, et al. Current applications of MRI-guided laser interstitial thermal therapy in the treatment of brain neoplasms and epilepsy: a radiologic and neurosurgical overview. AJNR Am J Neuroradiol 2015;36:1998-2006.

8. Gross RE, Willie JT, Drane DL. The role of stereotactic laser amygdalohippocampotomy in mesial temporal lobe epilepsy. Neurosurg Clin N Am 2016;27:37-50.

9. McNichols RJ, Gowda A, Kangasniemi M, Bankson JA, Price RE, Hazle JD. MR thermometry-based feedback control of laser interstitial thermal therapy at $980 \mathrm{~nm}$. Lasers Surg Med 2004;34:48-55.

10. Drane DL, Loring DW, Voets NL, et al. Better object recognition and naming outcome with MRI-guided stereotactic laser amygdalohippocampotomy for temporal lobe epilepsy. Epilepsia 2015;56:101-113.

11. Heck CN, King-Stephens D, Massey AD, et al. Two-year seizure reduction in adults with medically intractable partial onset epilepsy treated with responsive neurostimulation: final results of the RNS System Pivotal Trial. Epilepsia 2014;55:432-441.

12. Morrell MJ; RNS System in Epilepsy Study Group. Responsive cortical stimulation for the treatment of medically intractable partial epilepsy. Neurology 2011;77:1295-1304.

13. Bergey GK, Morrell MJ, Mizrahi EM, et al. Long-term treatment with responsive brain stimulation in adults with refractory partial seizures. Neurology 2015;84:810-817.

14. Eggleston KS, Olin BD, Fisher RS. Ictal tachycardia: the head-heart connection. Seizure 2014;23:496-505.

15. Boon P, Vonck K, van Rijckevorsel K, et al. A prospective, multicenter study of cardiac-based seizure detection to activate vagus nerve stimulation. Seizure 2015;32:52-61.

16. French JA, Krauss GL, Wechsler RT, et al. Perampanel for tonic-clonic seizures in idiopathic generalized epilepsy: a randomized trial. Neurology 2015;85:950-957.

17. Steinhoff BJ, Ben-Menachem E, Ryvlin P, et al. Efficacy and safety of adjunctive perampanel for the treatment of refractory partial seizures: a pooled analysis of three phase III studies. Epilepsia 2013;54: 1481-1489.

18. NDA 202834: FDA Approved Labeling Text for FYCOMPA [online]. Available at: http://www. accessdata.fda.gov/drugsatfda_docs/label/2012/202834lbl.pdf. Accessed May 4, 2016.

19. Maa E, Figi P. The case for medical marijuana in epilepsy. Epilepsia 2014;55:783-786.

20. Michoulam R, Shvo Y. Hashish: I: the structure of cannabidiol. Tetrahedron 1963;19:2073-2078.

21. Di Marzo V, Bifulco M, De Petrocellis L. The endocannabinoid system and its therapeutic exploitation. Nat Rev Drug Discov 2004;3:771-784.

22. Cilio MR, Thiele EA, Devinsky O. The case for assessing cannabidiol in epilepsy. Epilepsia 2014;55: 787-790.

23. Gross DW, Hamm J, Ashworth NL, Quigley D. Marijuana use and epilepsy: prevalence in patients of a tertiary care epilepsy center. Neurology 2004;62:2095-2097.

24. Keeler MH, Reifler CB. Grand mal convulsions subsequent to marijuana use: case report. Dis Nerv Syst 1967;28:474-475.

25. Gloss D, Vickrey B. Cannabinoids for epilepsy. Cochrane Database Syst Rev 2014;3:CD009270.

26. Devinsky O, Marsh E, Friedman D, et al. Cannabidiol in patients with treatment-resistant epilepsy: an open-label interventional trial. Lancet Neurol 2016;15:270-278.

27. GW Pharmaceuticals Announces Positive Phase 3 Pivotal Study Results of Epidiolex (cannabidiol) [online]. Available at: http://www.gwpharm.com/GW\%20Pharmaceuticals\%20Announces\% 20Positive\%20Phase\%203\%20Pivotal\%20Study\%20Results\%20for\%20Epidiolex\%20cannabidiol.aspx. Accessed May 4, 2016. 


\section{AUTHOR CONTRIBUTIONS}

Dr. Saipetch participated in study concept and design, and acquisition of data and manuscript preparation. Dr. Sachs participated in acquisition of data and manuscript preparation. Dr. Haneef provided study concept and design, manuscript preparation, and critical revision of the manuscript for intellectual content.

\section{STUDY FUNDING}

No targeted funding reported.

\section{DISCLOSURES}

C. Saipetch and E. Sachs report no disclosures. Z. Haneef has received funding for travel from NeuroPace and an honorarium from Lundbeck. Full disclosure form information provided by the authors is available with the full text of this article at Neurology.org/cp.

\section{Related articles from AAN physician and patient resources}

\section{Neurology ${ }^{\circledR}$ Clinical Practice}

Knowledge translation of an online tool to determine candidacy for epilepsy surgery evaluation

August 2016;6:304-314.

Diagnostic value of serum prolactin levels in PNES in the epilepsy monitoring unit April 2016;6:116-119.

\section{Continuum ${ }^{\circledR}$ • ContinuumJournal.com}

Diagnosis of Epilepsy and Related Episodic Disorders

February 2016;22:15-37.

\section{Neurology Today ${ }^{\circledR} \quad$ - Neurotodayonline.com}

News from the AAN Annual Meeting: Epilepsy Does Not Impact Fertility, New Study Finds April 21, 2016;16:1,18-21.

A Statistical Tool to Predict Outcomes with Epilepsy Surgery March 5, 2015;15:1,14-16.

INSIDE THE AAN SECTIONS: The Epilepsy Section - New Drugs, New Devices, and New Questions

February 5, 2015;15:17-21. 